Research Article

\title{
Quantitative Profiling of Oxylipin Reveals the Mechanism of Pien-Tze-Huang on Alcoholic Liver Disease
}

\author{
Ziye Zhu, ${ }^{1,2}$ Wenjun Zhou, ${ }^{1}$ Yang Yang, ${ }^{2}$ Kai Wang, ${ }^{2}$ Fenghua Li $\mathbb{D}^{2},{ }^{2}$ and Yanqi Dang $\mathbb{D}^{1}$ \\ ${ }^{1}$ Institute of Digestive Diseases, Longhua Hospital, China-Canada Center of Research for Digestive Diseases (ccCRDD), \\ Shanghai University of Traditional Chinese Medicine, Shanghai 200032, China \\ ${ }^{2}$ Experiment Center for Science and Technology, Shanghai University of Traditional Chinese Medicine, Shanghai 200032, China
}

Correspondence should be addressed to Fenghua Li; lfh@hotmail.com and Yanqi Dang; dangyanqi9022@126.com

Received 10 March 2021; Revised 30 April 2021; Accepted 6 May 2021; Published 1 June 2021

Academic Editor: Chan-Yen Kuo

Copyright (C) 2021 Ziye Zhu et al. This is an open access article distributed under the Creative Commons Attribution License, which permits unrestricted use, distribution, and reproduction in any medium, provided the original work is properly cited.

\begin{abstract}
Alcoholic liver disease (ALD) is a liver disease caused by long-term alcohol consumption. ROS-mediated oxidative stress is the leading cause of ALD. Pien-Tze-Huang (PZH), a traditional formula, is famous in China. This study was designed to evaluate the effects and explore the potential mechanisms of PZH in ALD. Forty mice were randomly divided into five groups: control group (normal diet + vehicle), model group (ethanol diet + vehicle), PZH-L group (ethanol diet + PZH (0.125 g/kg)), PZH-M group (ethanol diet $+\mathrm{PZH}(0.25 \mathrm{~g} / \mathrm{kg})$ ), and PZH-H group (ethanol diet $+\mathrm{PZH}(0.5 \mathrm{~g} / \mathrm{kg})$ ). The mice were sacrificed, and their liver and blood samples were preserved. Liver steatosis, triglyceride (TG), total cholesterol, serum alanine aminotransferase (ALT), and aspartate aminotransferase (AST) levels were assayed. Malondialdehyde (MDA), glutathione peroxidase (GSH-PX), and total superoxide dismutase were identified using commercial kits. Oxylipins were profiled, and the data were analyzed. The AMPK/ ACC/CPT1A pathway was identified using real-time polymerase chain reaction and western blotting. The PZH-H intervention significantly alleviated hepatic steatosis and injury and reduced the levels of liver TG and serum ALT and AST. In addition, MDA levels were markedly reduced, and GSH-PX activity significantly increased after PZH-H intervention. Finally, PZH-H increased the levels of 17-HETE, 15-HEPE, 9-HOTrE, 13-HOTrE, and 5,6-dihydroxy-8Z,11Z,14Z,17Z-eicosatetraenoic acid, and reduced PGE2 levels. PZH-H intervention also promoted the phosphorylation of AMPK and ACC, and the expression of CPT1A. In conclusion, PZH reduced oxidative stress and alleviated hepatic steatosis and injury. The mechanism was correlated with the oxylipin metabolites/AMPK/ACC/CPT1A axis.
\end{abstract}

\section{Introduction}

Alcoholic liver disease (ALD) is a liver disease caused by long-term alcohol consumption. It is one of the most common types of chronic liver diseases globally and is currently one of the major chronic liver diseases in China [1-3]. Heavy drinkers (ethanol consumption of $\geq 40 \mathrm{~g}$ /day for men and $\geq 20 \mathrm{~g} /$ day for women over 5 years; or ethanol consumption of $>80 \mathrm{~g} /$ day and binge drinking within 2 weeks) develop fatty liver, and about $20 \%-40 \%$ of them develop more severe ALD $[1,4]$. According to the International Classification of Diseases (ICD-10), ALD is classified as alcoholic fatty liver, alcoholic hepatitis, alcoholic liver fibrosis, alcoholic cirrhosis, and alcoholic liver failure. Studies have found that the prevalence of
ALD in China is 4.5\%, which is similar to that in European and American countries, among which the prevalence of ALD in people aged $40-49$ years is the highest, accounting for more than $10 \%$ [5]. At present, the effective ways to reduce or terminate alcoholic liver injury are nutritional support and abstinence from alcohol, but some patients still need to undergo combined treatment with drug intervention. The guidelines for the prevention and treatment in 2018 included glucocorticoid, metadoxine, S-adenosylmethionine, polyene phosphatidylcholine, glycyrrhizic acid preparation, and silymarin. Although these drugs show some improvement in patients with ALD, strict and extensive sample data supporting effective treatment and improvement of ALD are still lacking in clinical trials. 
Alcohol consumption can induce the generation of reactive oxygen species (ROS), which cause oxidative stress. ROS can change the structure and function of the protein by binding to them and finally generate host antigens that can induce immune responses [6, 7]. ROS can also lead to lipid peroxidation and generate lipid peroxidation products, such as 4-hydroxynonenal (4-HNE) and malondialdehyde (MDA). Studies have found that these products can bind to DNA bases and induce apoptosis and autophagy in cells [8-10]. Therefore, ROS-mediated oxidative stress is the leading cause of ALD.

Oxylipins are produced by the oxidation of polyunsaturated fatty acids (PUFAs), including arachidonic acid (AA), linoleic acid (LA), eicosapentaenoic acid (EPA), and docosahexaenoic acid (DHA). Oxylipins play an important role in inflammatory response $[11,12]$, immune defense $[13,14]$, and oxidative stress $[13,15]$. Studies have found that AA can inhibit inflammatory responses and oxidative stress to protect against brain injury [15]. LA attenuates acroleininduced oxidative stress [16]. EPA can decrease ROS to attenuate oxidative stress [17-19]. In addition, DHA protects photoreceptors from oxidative stress [20]. These studies have shown that oxylipins can attenuate oxidative stress.

The traditional formula, Pien-Tze-Huang (PZH), is famous in China and is mainly composed of four traditional Chinese medicines, namely, Panax notoginseng (85\%), Musk (3\%), Calculus bovis (5\%), and snake's gall bladder (7\%). Studies have found that PZH has the functions of clearing away heat and detoxification, reducing swelling and relieving pain, anti-inflammatory effects, and liver protection [21-23]. Clinically, it is widely used in various inflammatory diseases, such as viral hepatitis, alcoholic hepatitis, and fatty liver [24]. Currently, the mechanism of PZH in ALD remains unclear. Therefore, in this study, we explored the protective effect of PZH on ALD and its potential mechanism.

\section{Materials and Methods}

2.1. Animals. C57BL/6 male mice (22-25g) were obtained from Beijing Vital River Laboratory Animal Technology Co. Ltd, China. Mice were maintained under a specific pathogen-free environment $\left(23-25^{\circ} \mathrm{C}\right.$, relative humidity: $40 \%-$ $70 \%$ ) with a 12 -hour light/dark cycle.

2.2. Experimental Design and Administration of PZH. Mice were randomly divided into five groups: control group (normal diet + vehicle), model group (ethanol die$\mathrm{t}+$ vehicle), PZH-L group (ethanol diet + PZH $(0.125 \mathrm{~g} / \mathrm{kg})$ ), $\mathrm{PZH}-\mathrm{M}$ group (ethanol diet + PZH $(0.25 \mathrm{~g} / \mathrm{kg})$ ), and PZH-H group (ethanol diet $+\mathrm{PZH}(0.5 \mathrm{~g} / \mathrm{kg})$ ). Mice fed an ethanol diet were fed an ethanol Lieber-DeCarli diet (Trophic Animal Feed High-tech Co. Ltd, China) containing 5\% (v/v) ethanol for 10 days. Mice with a normal diet were pair-fed with an equicaloric normal Lieber-DeCarli diet for 10 days. PZH was exclusively produced and certified by Zhangzhou Pien Tze Huang Pharmaceutical Co., Ltd., and the fingerprint spectrum was determined by HPLC-MS in a previous study [22]. PZH-treated mice were gavaged with $\mathrm{PZH}$ aqueous solution simultaneously as they were fed an ethanol diet for 10 days. On day 11, mice fed an ethanol diet were gavaged with a single dose of $31.5 \%(\mathrm{v} / \mathrm{v})$ ethanol $(20 \mu \mathrm{L} / \mathrm{g}$ body weight), and mice fed a normal diet were gavaged with equicaloric $45 \%(\mathrm{w} / \mathrm{v})$ maltose dextrin $(20 \mu \mathrm{L} / \mathrm{g}$ body weight). All mice were euthanized after 9 hours, and blood and liver samples were collected for further study.

2.3. Biochemical Analysis. The blood was centrifuged for $20 \mathrm{~min}$ at $4000 \mathrm{rpm}$ at $4^{\circ} \mathrm{C}$, and serum was collected for analysis. Liver tissue was homogenized in propanone/ethanol $(1: 1)$ and incubated overnight at $4^{\circ} \mathrm{C}$. Homogenates were centrifuged for $10 \mathrm{~min}$ at $4000 \mathrm{rpm}$ at $4^{\circ} \mathrm{C}$, and the supernatant was collected for testing. Serum alanine aminotransferase (ALT) and aspartate aminotransferase (AST) levels, liver triglyceride (TG), and total cholesterol (TC) levels were measured using an automatic biochemistry analyzer (TBA-40FR, Toshiba, Japan).

2.4. Histological Analysis. The left lobe of the liver was cut in half. One half was fixed in $10 \%$ buffered formalin, and the other was frozen in liquid nitrogen. The fixed liver sections were dehydrated, embedded, and sectioned into $4 \mu \mathrm{m}$ sections for staining with hematoxylin and eosin $(\mathrm{H} \& \mathrm{E})$. The frozen liver sections were embedded and sectioned into $10 \mu \mathrm{m}$ thick sections using a frozen microtome (Leica, Germany) for staining with Oil Red O, according to previous studies $[25,26]$.

2.5. Measurement of MDA, GSH-PX, and T-SOD Levels in the Liver. Liver tissue was homogenized and centrifuged for $10 \mathrm{~min}$ at $4000 \mathrm{rpm}$ at $4^{\circ} \mathrm{C}$, and the supernatant was collected. The levels of MDA, glutathione peroxidase (GSH$\mathrm{PX}$ ), and total superoxide dismutase (T-SOD) in liver tissue were measured using commercial kits (Nanjing Jiancheng Bioengineering Institute, Nanjing, China).

2.6. Sample Preparation and the Detection of Oxylipins Profile. Tissue $(20 \mathrm{mg})$ was weighed and homogenized with $200 \mathrm{~L}$ of oxylipin extract (BB24, Next Advance, Inc., Averill Park, NY, USA) for $3 \mathrm{~min}$. The mixture was centrifuged for $10 \mathrm{~min}$ at $5000 \mathrm{rpm}$ at $4^{\circ} \mathrm{C}$. The supernatant was concentrated, dried, and then redissolved in $100 \mathrm{~L}$ methanol/water $(1: 1, \mathrm{v} / \mathrm{v})$. Seventy-one oxylipin metabolites were determined at the Metabo-Profile R \& D Laboratory (Shanghai, China). Ultraperformance liquid chromatography-tandem mass spectrometry (UPLC-MS/MS) (ExionLC ${ }^{\text {TM }}$ AD, QTRAP ${ }^{\circledR} 6500+$ ) was used to detect them according to manufacturer's instructions.

2.7. Oxylipins Data Analysis. The raw data generated by UPLC-MS/MS were extracted, integrated, identified, and quantitatively analyzed for each metabolite using AB Sciex's Analyst software (V1.6.3, AB Sciex, Boston, MA, USA). Principal component analysis (PCA), partial least squares 
discriminant analysis (PLS-DA), and variable importance of projection (VIP) were performed to analyze the data. The formula used for calculating the $Z$-score was $Z$-scores = (x-mean)/SD. The Euclidean distance of Z-scores was clustered with the method in the heatmap. Kyoto Encyclopedia of Genes and Genomes (KEGG) pathways were enriched using Metaboanalyst 4.0.

2.8. Real-Time Polymerase Chain Reaction (RT-PCR). Total RNA was isolated from liver tissue using RNAiso Reagent (Takara, Japan) and reverse-transcribed using PrimeScript ${ }^{\mathrm{TM}}$ RT Master Mix (Takara, Japan). RT-PCR was performed using TB Green ${ }^{\circledR}$ Premix Ex Taq ${ }^{\mathrm{TM}}$ according to the manufacturer's instructions. Target gene expression was analyzed using the $2^{-\Delta \Delta \mathrm{Ct}}$ method by normalization to $\beta$-actin. The primers used are listed in Table 1.

2.9. Western Blot Analysis. Liver tissue was lysed and centrifuged for $10 \mathrm{~min}$ at $4000 \mathrm{rpm}$ at $4^{\circ} \mathrm{C}$, and the supernatant was collected and denatured. Denatured proteins were separated by SDS-PAGE and transferred to polyvinylidene fluoride membranes (Millipore, USA). The membranes were blocked using 5\% skim milk and then incubated at $4^{\circ} \mathrm{C}$ overnight with the primary antibodies, including AMP-activated protein kinase alpha $(\mathrm{AMPK} \alpha)$ (CST, USA), phospho-AMPK $\alpha$ (CST, USA), acetyl-CoA carboxylase (ACC) (CST, USA), phospho-ACC (CST, USA), carnitine palmitoyltransferase 1A (CPT1A) (Gene Tex), peroxisome proliferator-activated receptor alpha (PPAR $\alpha)$ (ABclonal, China), sterol regulatory element-binding transcription factor 1 (SREBP1) (Santa Cruz, USA), and HRPconjugated $\beta$-actin (HUABIO, China). After the overnight incubation, the membranes were incubated with HRPconjugated secondary antibody (Jackson Immuno). Blots were visualized with ECL luminescence reagent (Meilunbio, China) and quantified using ImageJ software.

2.10. Statistical Analysis. Data are presented as mean\pm standard deviation $(\bar{x} \pm s)$. Statistical significance was analyzed using one-way ANOVA in SPSS 24.0. Qualitative data among the three groups were assessed using one-way ANOVA, the Kruskal-Wallis test, or the chi-square test based on data distribution. Differences between the two groups were analyzed using Student's $t$-test. The values were considered statistically significant at $P<0.05$.

\section{Results}

3.1. PZH-H Alleviated Hepatic Steatosis in ALD-Mice. Hepatic steatosis was significantly higher in the model group than in the control group, and the $\mathrm{PZH}-\mathrm{M}$ and $\mathrm{PZH}-\mathrm{H}$ interventions significantly improved hepatic steatosis (Figures 1(a) and 1(b)). In addition, liver TG and TC levels were markedly increased in the model group compared with those in the control group. The $\mathrm{PZH}-\mathrm{H}$ intervention significantly reduced liver TG levels, but liver TC was increased in the PZH-H group compared with that in the model group
(Figures 1(c) and 1(d)). Furthermore, serum ALT and AST levels were markedly increased in the model group compared with those in the control group. The PZH-H intervention markedly reduced ALT and AST levels, and the PZH-M intervention also markedly reduced ALT levels (Figures 1(e) and 1(f)). These results indicated that PZH-M and $\mathrm{PZH}-\mathrm{H}$ alleviated hepatic steatosis and injury in ALD mice, and the effect of $\mathrm{PZH}-\mathrm{H}$ was better than that of $\mathrm{PZH}-$ M.

3.2. Effect of PZH on MDA, GSH-PX, and SOD. Oxidative stress is one of the main pathogeneses of ALD; MDA, GSH$\mathrm{PX}$, and T-SOD are the primary biomarkers of oxidative stress. Thus, we tested the levels of MDA, GSH-PX, and T-SOD in the livers of ALD-mice. Compared with the control group, the level of MDA in the model group was significantly increased, while the activities of GSH-PX and T-SOD were significantly decreased. Compared with the model group, the level of MDA in the three PZH groups was significantly decreased (Figure 2(a)), and the activity of GSH-PX in the PZH-H group was significantly increased (Figure 2(b)). Although T-SOD activity in the PZH-H group was not different, its activity was significantly increased in the PZH-M group (Figure 2(c)). These results indicate that $\mathrm{PZH}-\mathrm{H}$ could improve oxidative stress.

\subsection{Analysis of Oxylipin Metabolites among Three Groups.} Our results showed that $\mathrm{PZH}$ could alleviate hepatic steatosis, liver injury, and oxidative stress, and the effect of $\mathrm{PZH}$ $\mathrm{H}$ was better than that of the PZH-L and PZH-M groups. Thus, we chose the $\mathrm{PZH}-\mathrm{H}$ group to explore the mechanism of oxidative stress improvement. Previous studies have proved that oxylipins play an important role in oxidative stress [13, 15]. Therefore, the profiling of oxylipins was performed and analyzed. The results indicated that the proportion of DHA was the highest, followed by that of LA (Figure 3(a)). DHA, LA, and EPA levels were significantly different among the three groups (Figure 3(b)). Fifty-eight oxylipin metabolites were detected in the liver tissue of the mice (Figure 3(c), Supplementary file 1). The PCA and PLSDA analyses yielded clear distinctions among the three groups (Figures 3(d) and 3(e)).

3.4. Effect of PZH on Different Oxylipin Metabolites. First, using values of $P<0.05$, the sets of 24 and 16 different oxylipin metabolites were obtained in the model group compared with the control group, and the $\mathrm{PZH}-\mathrm{H}$ group compared with the model group (Figures $4(\mathrm{a})$ and $4(\mathrm{c})$ ), respectively. Furthermore, combined with the value of VIP $>1$, only 22 and 16 different oxylipin metabolites were obtained in the model group compared with the control group, and the $\mathrm{PZH}-\mathrm{H}$ group compared with the model group (Figures 4(b) and 4(d), Supplementary file 2), respectively. KEGG pathway analysis revealed that the main pathways included carnitine $\mathrm{O}$-acetyltransferase, carnitineacetylcarnitine carrier, fatty acyl-CoA desaturase, linoleic acid (n-C18:2) transport via diffusion, carnitine 
TABLE 1: The sequence of primers in the study.

\begin{tabular}{lll}
\hline Primer name & Forward primer sequence $\left(5^{\prime} \longrightarrow 3^{\prime}\right)$ & Reverse primer sequence $\left(5^{\prime} \longrightarrow 3^{\prime}\right)$ \\
\hline$\beta$-Actin & GGCTGTATTCCCCTCCATCG & CCAGTTGGTAACAATGCCATGT \\
ACC1 & GATGAACCATCTCCGTTGGC & GACCCAATTATGAATCGGGAGTG \\
ACC2 & CCTTTGGCAACAAGCAAGGTA & AGTCGTACACATAGGTGGTCC \\
CPT1A & CTCCGCCTGAGCCATGAAG & CACCAGTGATGATGCCATTCT \\
PPAR $\alpha$ & AGAGCCCCATCTGTCCTCTC & ACTGGTAGTCTGCAAAACCAAA \\
SREBP-1 & GCAGCCACCATCTAGCCTG & CAGCAGTGAGTCTGCCTTGAT \\
\hline
\end{tabular}

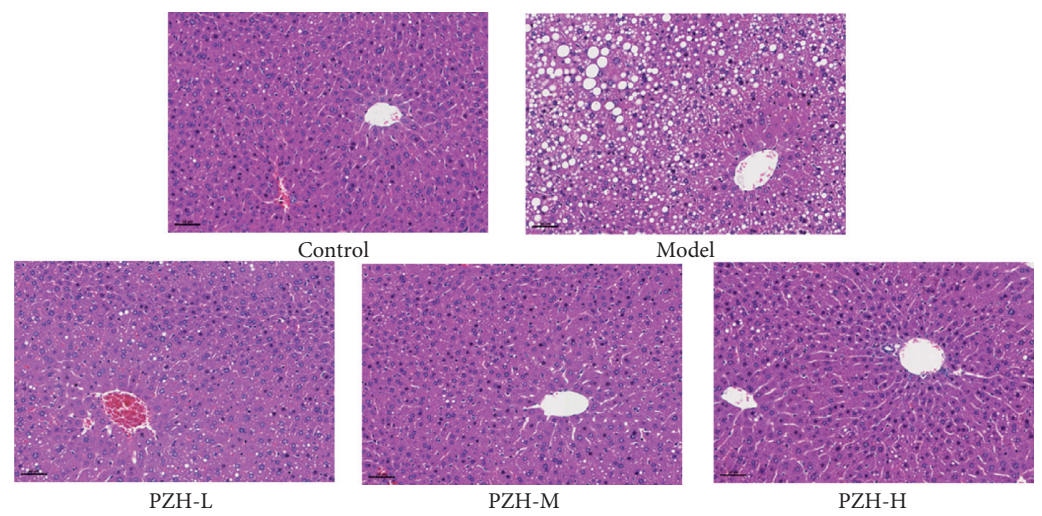

(a)

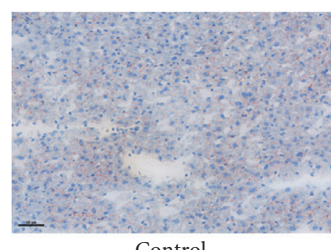

Control

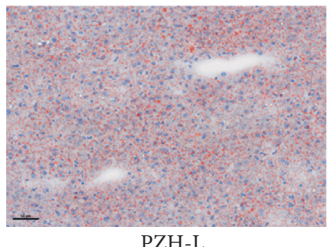

PZH-L

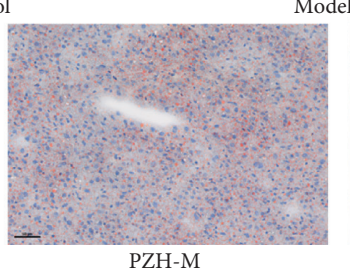

(b)

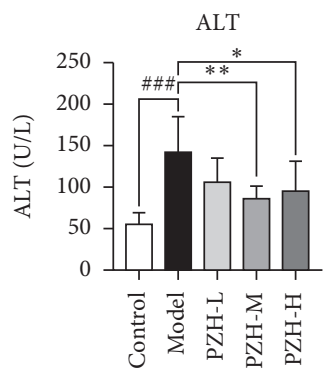

(e)

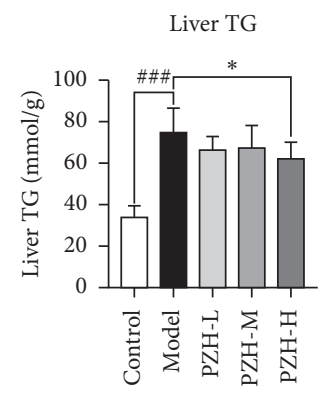

(c)

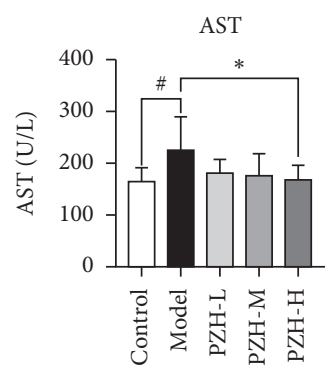

(f)

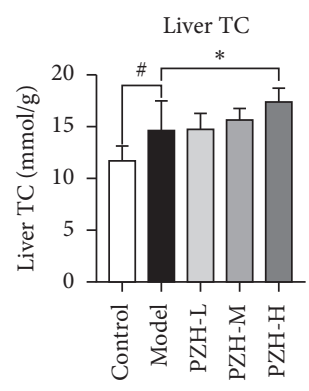

(d)

Figure 1: PZH-H alleviated hepatic steatosis in ALD-mice. (a) HE staining in liver sections was presented. (b) Oil Red O in liver sections was presented. (c) Liver TG and (d) TC were analyzed $(n=8)$; (e) Serum ALT and (f) AST were analyzed $(n=8)$. Data were presented as means \pm SD. ${ }^{\#} P<0.05,{ }^{\# \#} P<0.01$, and ${ }^{\# \# \#} P<0.01$ in the model group vs the control group; ${ }^{*} P<0.05$ and ${ }^{* *} P<0.01$ in the PZH groups vs the model group.

O-palmitoyltransferase, and beta-oxidation of fatty acids (Figures 4(e) and 4(f)). We further identified six overlapping oxylipin metabolites among the three groups using a Venn diagram (Figure 5(a)), including two EPAs (17-HETE and 15-HEPE), three LAs (9-HOTrE, 13-HOTrE, 5,6-dihydroxy$8 \mathrm{Z}, 11 \mathrm{Z}, 14 \mathrm{Z}, 17 \mathrm{Z}$-eicosatetraenoic acid), and one DGLA 


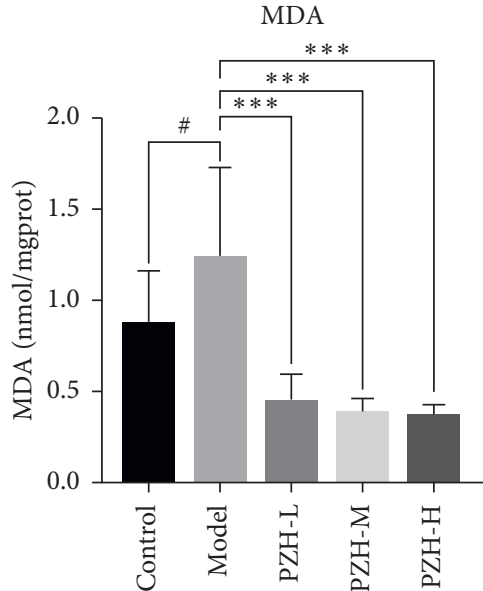

(a)

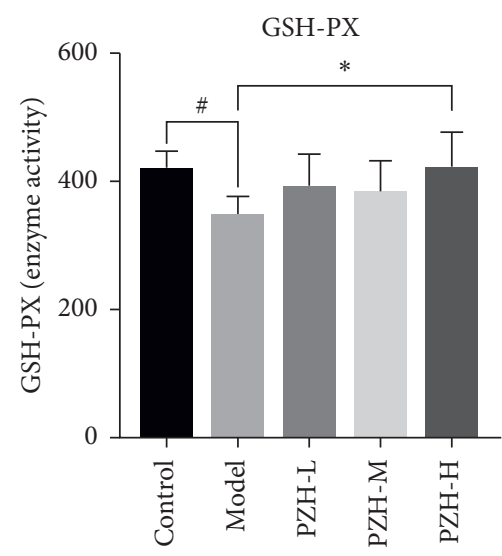

(b)

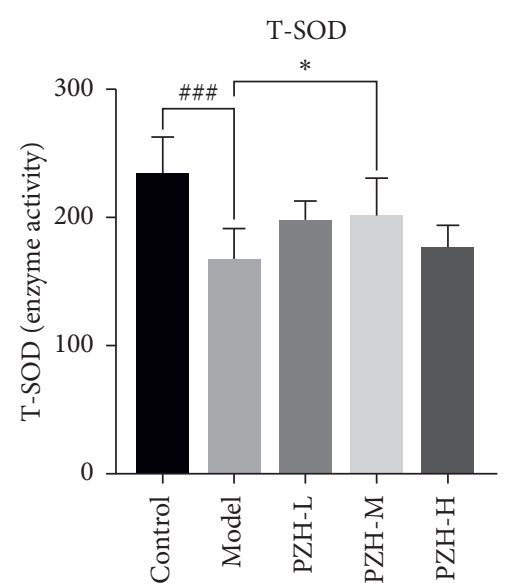

(c)

Figure 2: Effect of PZH on MDA, GSH-PX, and T-SOD. (a) The content of MDA, (b) the activity of GSH-PX, and (c) T-SOD was measured using kits $(n=8)$. Data were presented as means \pm SD. ${ }^{\#} P<0.05$ and ${ }^{\# \# \#} P<0.001$ in the model group vs the control group; ${ }^{*} P<0.05$ and ${ }^{* * *} P<0.001$ in the $\mathrm{PZH}$ groups vs the model group.

(PGE2). The levels of 17-HETE, 15-HEPE, 5,6-dihydroxy8Z, 11Z, 14Z, 17Z-eicosatetraenoic acid, 9-HOTrE, and 13HOTrE were markedly increased, and PGE2 levels were significantly reduced in the model group compared with the control group. $\mathrm{PZH}-\mathrm{H}$ intervention further increased the levels of 17-HETE, 15-HEPE, 9-HOTrE, 13-HOTrE, and 5,6dihydroxy-8Z, 11Z, 14Z, 17Z-eicosatetraenoic acid, and reduced PGE2 levels (Figures 5(b)-5(g), Table 2).

3.5. Oxylipin Metabolites Were Negatively Correlated with $M D A$. PZH-H reduced MDA levels and promoted the activity of GSH-PX (Figure 2). Profiling of oxylipins indicated that PZH-H could also increase the levels of 17-HETE, 15HEPE, 5,6-dihydroxy-8Z, 11Z, 14Z, 17Z-eicosatetraenoic acid, 9-HOTrE, 13-HOTrE, and reduced PGE2 levels (Figure 4). Therefore, Spearman correlation analysis was performed to evaluate the correlation of oxylipin metabolites, MDA, and GSH-PX. The results showed that 15-HEPE, 5,6dihydroxy-8Z, 11Z, 14Z, 17Z-eicosatetraenoic acid, 9HOTrE, and 13-HOTrE were negatively correlated with $\mathrm{MDA}$, and there was no correlation among the other indices (Figure 6).

3.6. Effect of PZH on AMPK-ACC-CPT1A Pathway. Previous studies have indicated that oxylipin metabolites can regulate the AMPK signaling pathway [27-30]. The results showed that although the phosphorylation of AMPK was not significantly different in the model group compared with the control group, $\mathrm{PZH}-\mathrm{H}$ intervention markedly promoted the phosphorylation of AMPK (Figure 7(a)). In addition, compared with the control group, the phosphorylation of ACC was significantly reduced in the model group, and $\mathrm{PZH}-\mathrm{H}$ intervention markedly promoted the phosphorylation of ACC. However, the mRNA levels of ACC1 and ACC2 in the model group were significantly increased, the mRNA levels of ACC1 were significantly decreased, and
ACC2 levels were significantly increased after PZH-H intervention (Figures $7(\mathrm{a})-7(\mathrm{c})$ ). Compared with the control group, the mRNA and protein levels of CPT1A were significantly decreased in the model group, and $\mathrm{PZH}-\mathrm{H}$ intervention significantly promoted CPT1A expression (Figures 7(a) and 7(d)). Although the phosphorylation of $\operatorname{PPAR} \alpha$ was not significantly different in the model group compared with the control group, the $\mathrm{PZH}-\mathrm{H}$ intervention markedly promoted the phosphorylation of PPAR $\alpha$. Moreover, the expression of SREBP1 was not significantly different among the three groups (Figures $7(\mathrm{e})-7(\mathrm{~g})$ ).

\section{Discussion}

In the present study, we showed that PZH could improve hepatic steatosis and injury in ALD mice, consistent with previous studies. In addition, $\mathrm{PZH}$ reduced the level of MDA and increased the activity of GSH-PX, ultimately ameliorating oxidative stress, which is consistent with a previous study [31]. Through the analysis of oxylipin profiling, we found that PZH promoted the levels of 17-HETE, 15-HEPE, 5,6-dihydroxy-8Z, 11Z, 14Z, 17Z-eicosatetraenoic acid, 9HOTrE, and 13-HOTrE, and reduced PGE2 levels, further activating the AMPK pathway. Previous studies have also shown that $\mathrm{PZH}$ can reduce tumor necrosis factor-alpha and interleukin- $1 \beta$ secretion to ameliorate hepatic inflammation [31] and can ameliorate hepatic injury by inhibiting the PERK/eIF2 $\alpha$ pathway [22]. In addition, PZH could also ameliorate hepatic fibrosis by suppressing the NF-kappa B pathway [21]. Panax notoginseng saponins and polysaccharides have hepatoprotective effects against alcoholic liver damage [32, 33], but the mechanism is not precise. Our study is the first to demonstrate that PZH regulates the oxylipin-metabolites/AMPK pathway to reduce oxidative stress and improve hepatic steatosis and injury in ALD-mice. This study fully confirmed the mechanism of action of PZH on ALD through the quantitative profiling of oxylipin. 

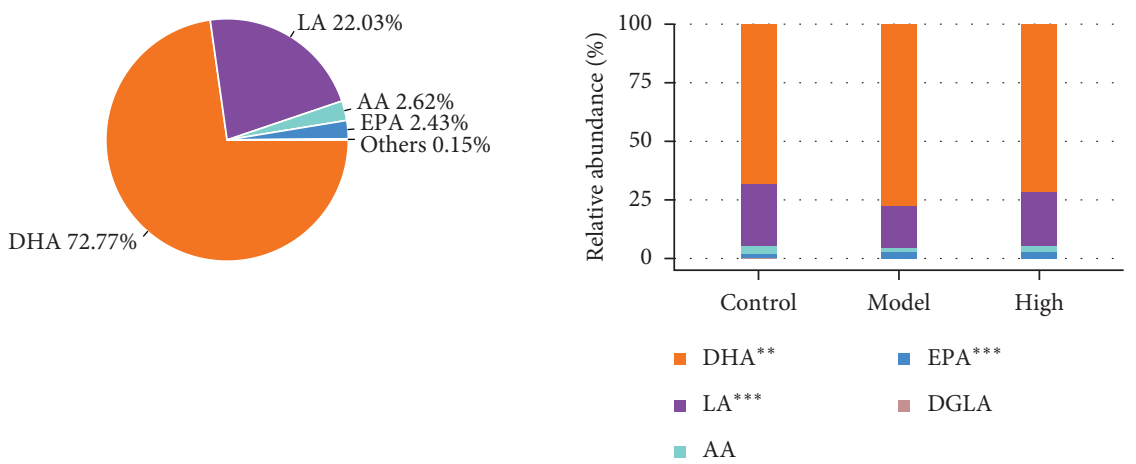

(a)

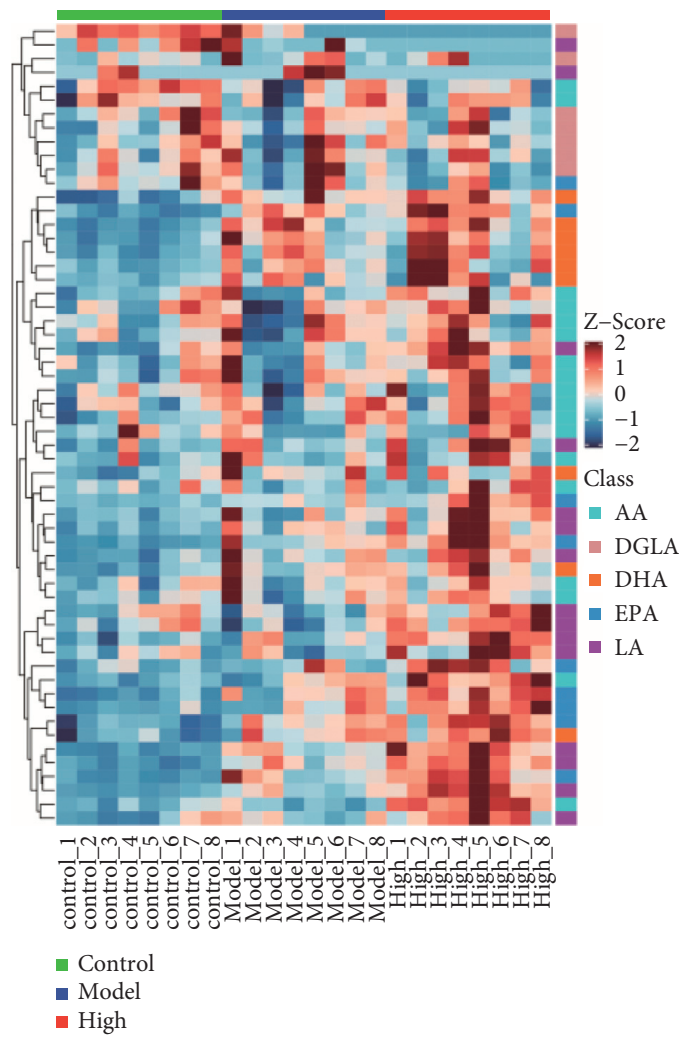

(b)
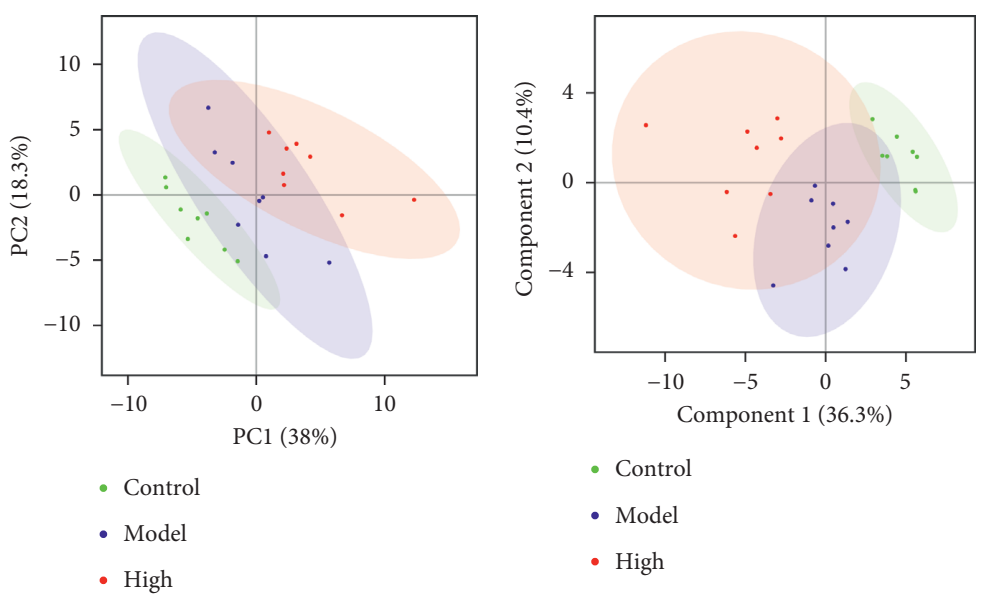

- Control

- Model

- High

(c)

(d)

(e)

FIGURE 3: Analysis of oxylipin metabolites among three groups. (a) The proportion of identified metabolite classes in all groups was shown. (b) The relative abundance of each metabolite class in different groups was shown. (c) Fifty-eight oxylipin metabolites were detected in the liver tissue of mice. (d) PCA and (e) PLS-DA were analyzed and shown. High: PZH-H group.

Oxidative stress is one of the main pathogeneses of ALD. As a primary biomarker of oxidative stress, MDA was markedly increased in ALD and promoted the generation of etheno-DNA adducts to induce damage to the liver $[9,10]$. In the present study, MDA levels were also markedly increased in the ALD mice, and PZH intervention significantly reduced MDA levels. In addition, the activity of GSH-PX, an antioxidant enzyme, was significantly reduced in ALD-mice, consistent with a previous study [34], and PZH increased the activity. These results indicated that PZH could attenuate oxidative stress.

Oxylipins play an essential role in oxidative stress $[13,15]$. Studies have shown that EPA, as an oxylipin, can decrease oxidative stress and placental lipid deposition in a sirtuin-1-independent manner [17]. The EPA could also decrease oxidative stress and attenuate cardiomyoblast apoptosis by activating autophagy [35]. EPA supplementation attenuates HFD-induced oxidative stress and steatosis [36]. In this study, PZH promoted the levels of EPA (17-HETE, 15-HEPE). Furthermore, LA is also an oxylipin, and studies have shown that LA can attenuate acrolein-induced oxidative stress [16]. Clinical trials have confirmed that LA can improve oxidative stress in obese patients with non-alcoholic fatty liver disease [37]. In our study, PZH increased LA levels, including 9-HOTrE, 13-HOTrE, and 5,6-dihydroxy$8 Z, 11 Z, 14 Z, 17 Z$-eicosatetraenoic acid. Studies have also 


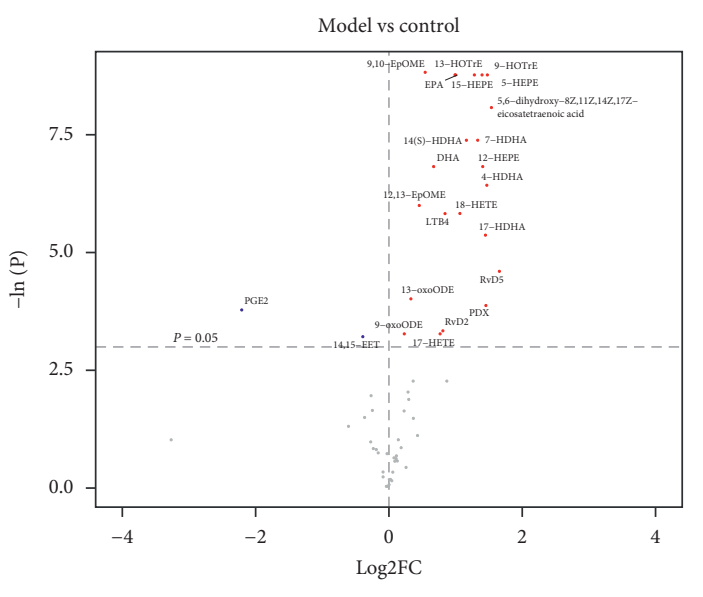

- Up:22

- Down:2

- None:34

(a)

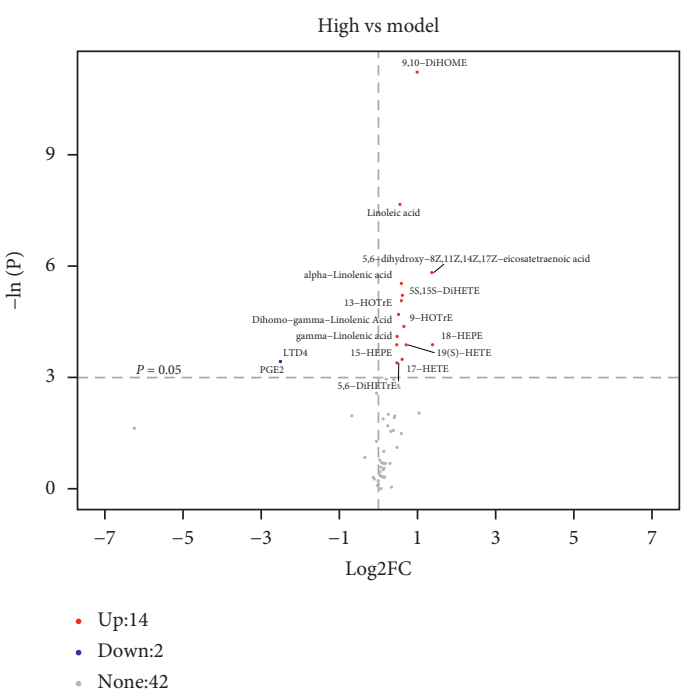

(c)

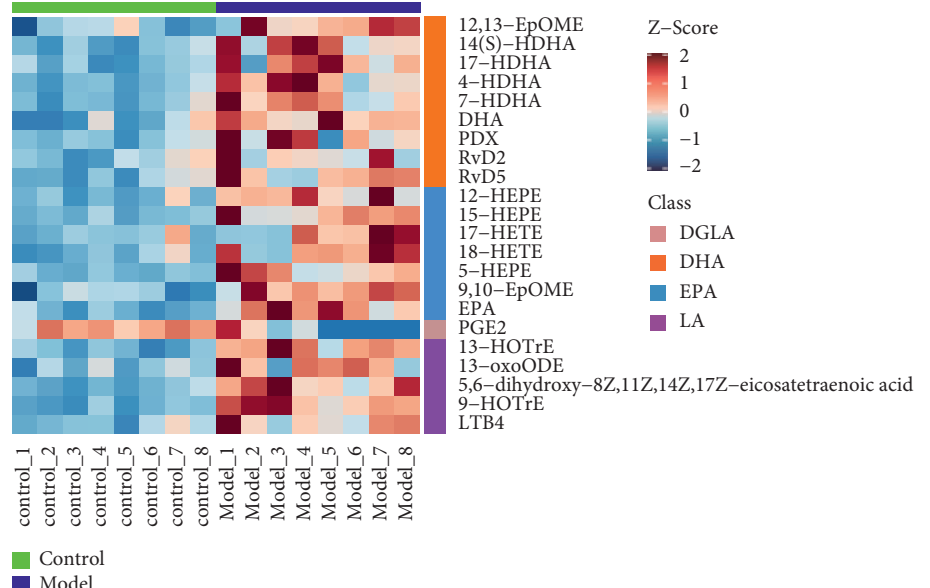

(b)

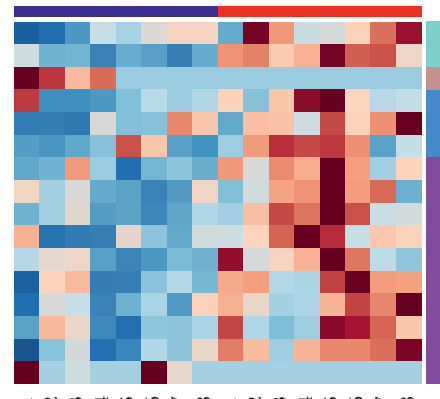

19(S)-HETE

9,10-DiHOME

PGE2

$15-\mathrm{HEPE}$

17-HETE

18-HEPE

13-HOTrE

5,6-DiHETrE

5,6-dihydroxy-8Z,11Z,14Z,17Z-eicosatetraenoic acid

5S,15S-DiHETE

9-HOTrE

AA

Dihomo-gamma-Linolenic Acid DGLA

Gamma-Linolenic acid
Linoleic acid

Linoleic acid
LTD4

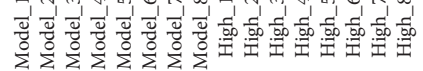

- Model

High

(d)

Figure 4: Continued. 


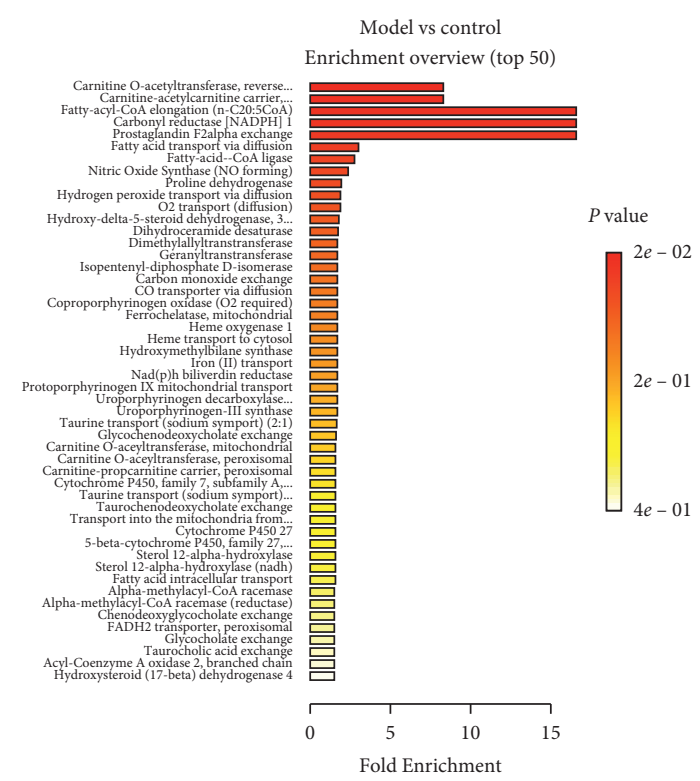

(e)

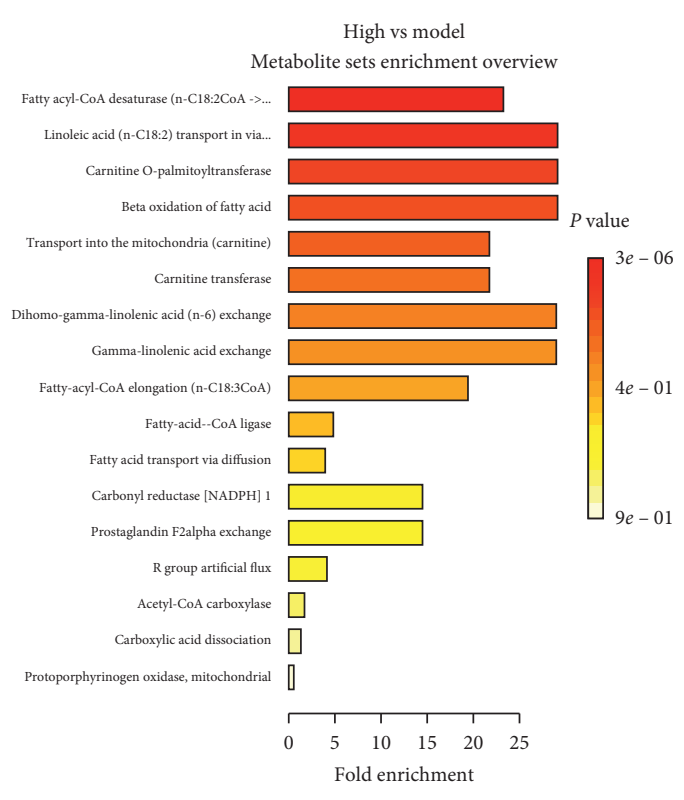

(f)

FIgUre 4: Effect of PZH on different oxylipin metabolites. (a) At $P<0.05,24$ different oxylipin-metabolites were obtained in the model group compared with the control group. (b) Combined with the value of VIP $>1,22$ different oxylipin metabolites were obtained in the model group compared with the control group. (c) At $\mathrm{P}<0.05,16$ different oxylipin-metabolites were obtained in the PZH-H group compared with the model group. (d) Combined with the value of VIP $>1,16$ different oxylipin metabolites were obtained in the PZH-H group compared with the model group. The KEGG pathway was shown in (e) the model group compared with the control group and (f) the PZH-H group compared with the model group. High: PZH-H group.

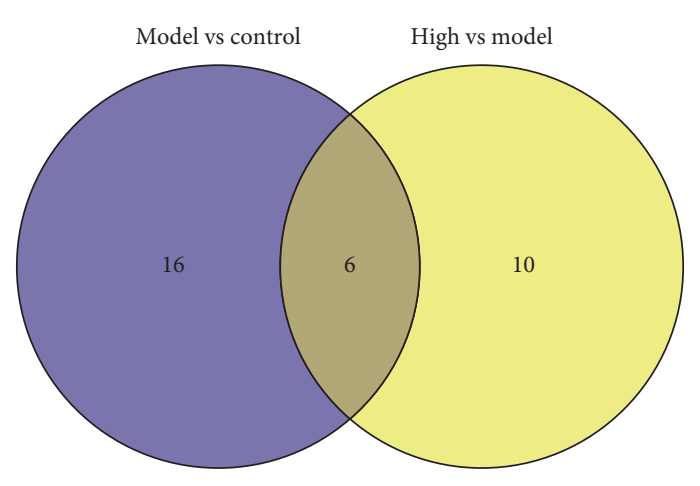

(a)

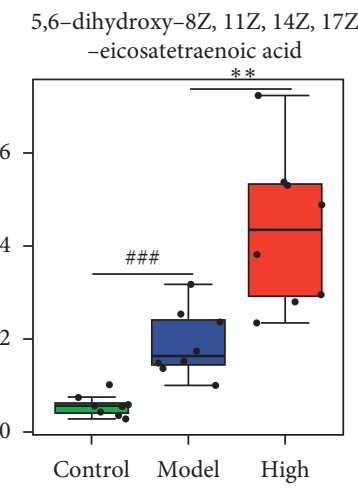

(d)

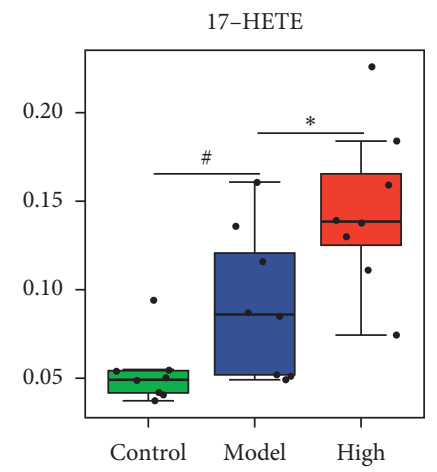

(b)

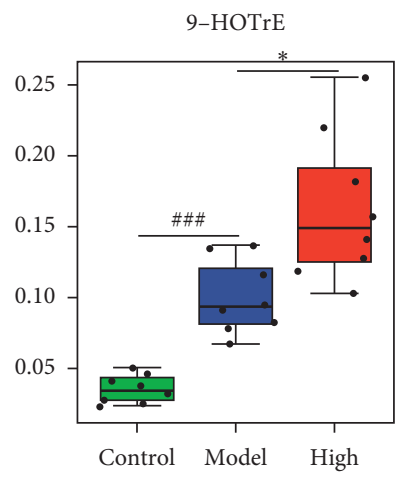

(e)

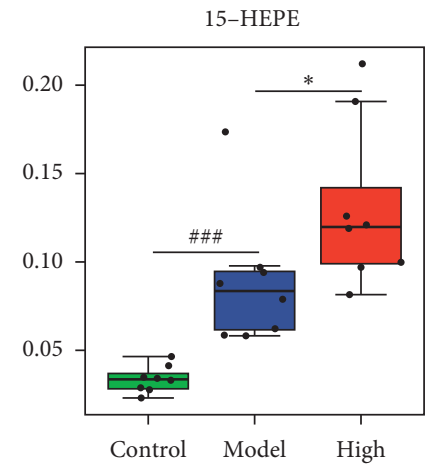

(c)

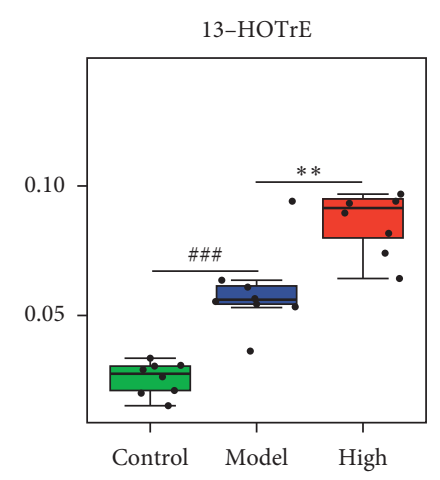

(f)

Figure 5: Continued. 


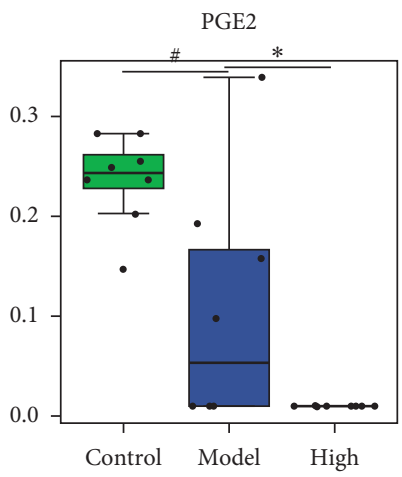

(g)

FIGURE 5: Effect of PZH on 6 overlapping oxylipin metabolites. (a) 6 overlapping oxylipin metabolites were showed among three groups. (b) 17-HETE, (c) 15-HEPE, (d) 5,6-dihydroxy-8Z,11Z,14Z,17Z-eicosatetraenoic acid, (e) 9-HOTrE, (f) 13-HOTrE, and (g) PGE2 levels were shown. ${ }^{\#} P<0.05$ and ${ }^{\# \# \#} P<0.001$ in the model group vs the control group; ${ }^{*} P<0.05$ and ${ }^{* *} P<0.01$ in the PZH group vs the model group. High: PZH-H group.

TABLE 2: Six overlapping oxylipin metabolites among three groups.

\begin{tabular}{|c|c|c|c|c|c|c|c|}
\hline \multirow{2}{*}{ Class } & \multirow{2}{*}{ Metabolite } & \multicolumn{2}{|c|}{ Model vs. control } & \multicolumn{4}{|c|}{ PZH-H vs. model } \\
\hline & & Fold change & $P$ value & VIP & Fold change & $P$ value & VIP \\
\hline EPA & 17-HETE & 1.735887097 & 0.037917638 & 1.059880581 & 1.574488 & 0.030620 & 1.438892 \\
\hline EPA & 15-HEPE & 2.479289941 & 0.0001554 & 1.470827013 & 1.431981 & 0.020668 & 1.013007 \\
\hline LA & $\begin{array}{c}\text { 5,6-Dihydroxy-8Z, } 11 Z, 14 Z, 17 Z \text {-eicosatetraenoic } \\
\text { acid }\end{array}$ & 2.960944596 & 0.0003108 & 1.678973419 & 2.665644 & 0.002953 & 1.914047 \\
\hline LA & 9-HOTrE & 2.685344828 & 0.0001554 & 1.810016764 & 1.625732 & 0.012600 & 1.562081 \\
\hline LA & 13-HOTrE & 2.027124774 & 0.0001554 & 1.770831487 & 1.554011 & 0.006302 & 1.812153 \\
\hline DGLA & PGE2 & 0.220679012 & 0.022819743 & 1.13067223 & 0.181818 & 0.032474 & 1.211364 \\
\hline
\end{tabular}

shown that high DGLA levels could be a biomarker for hepatic steatosis [38]. PGE2 can promote hepatic fibrosis and lead to insulin resistance [39]. Green tea extract attenuated PGE2 accumulation and protected against liver injury in an HFD-feeding NASH model [40], and meloxicam reduced PGE2 level and modulated oxidative stress to protect against liver injury [41]. Moreover, Panax notoginseng saponins attenuated gastric injury by modulating the metabolism of PGE2 [42]. In the present study, we found that $\mathrm{PZH}$ reduced $\mathrm{PGE} 2$ levels. In addition, our results further proved that 15-HEPE, 9-HOTrE, 13-HOTrE, and 5,6-dihydroxy-8Z, 11Z, 14Z, 17Z-eicosatetraenoic acid were negatively correlated with MDA. Further analysis showed that the upregulated metabolites (15-HEPE, 5,6-dihydroxy$8 \mathrm{Z}, 11 \mathrm{Z}, 14 \mathrm{Z}, 17 \mathrm{Z}$-eicosatetraenoic acid, 9-HOTrE, and 13-HOTrE) were negative correlated with T-SOD, and downregulated PGE2 were positively correlated with T-SOD (Supplementary Figure 1), indicating that $\mathrm{PZH}$ could reduce oxidative stress through non-SOD dependent approach. Finally, the results showed that $\mathrm{PZH}$ may improve oxidative stress by oxylipin metabolites, including EPA, LA, and PGE2. On the basis of a previous study [42], we speculated that Panax notoginseng of PZH may mainly enhance oxylipin metabolites.

The AMPK-ACC-CPT1A pathway is involved in the regulation of oxidative stress and ALD [43-47]. Previous

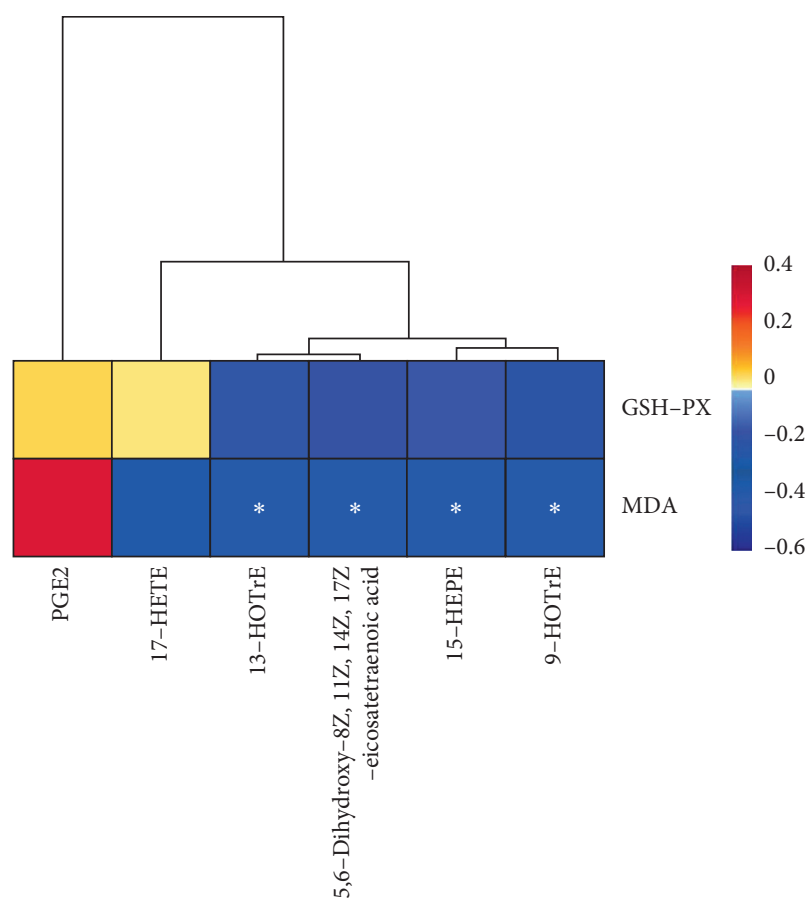

FIGURE 6: The correlation of oxylipin-metabolites, MDA, and GSHPX was analyzed by the Spearman correlation analysis. 


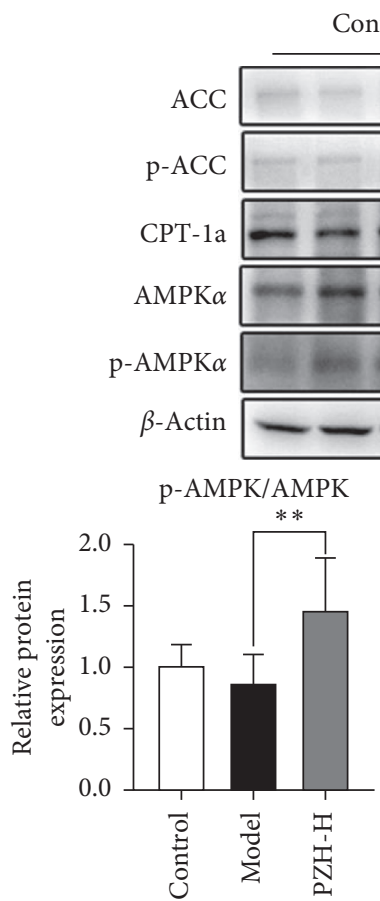

Control

Model

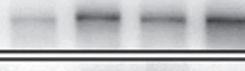

$\longrightarrow$
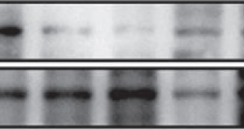

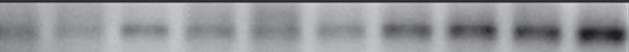

$-1---1$

p-ACC/ACC

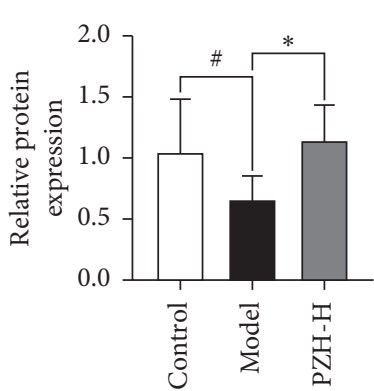

(a)

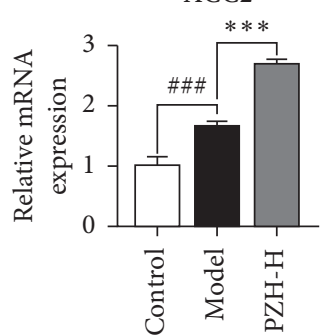

(c)
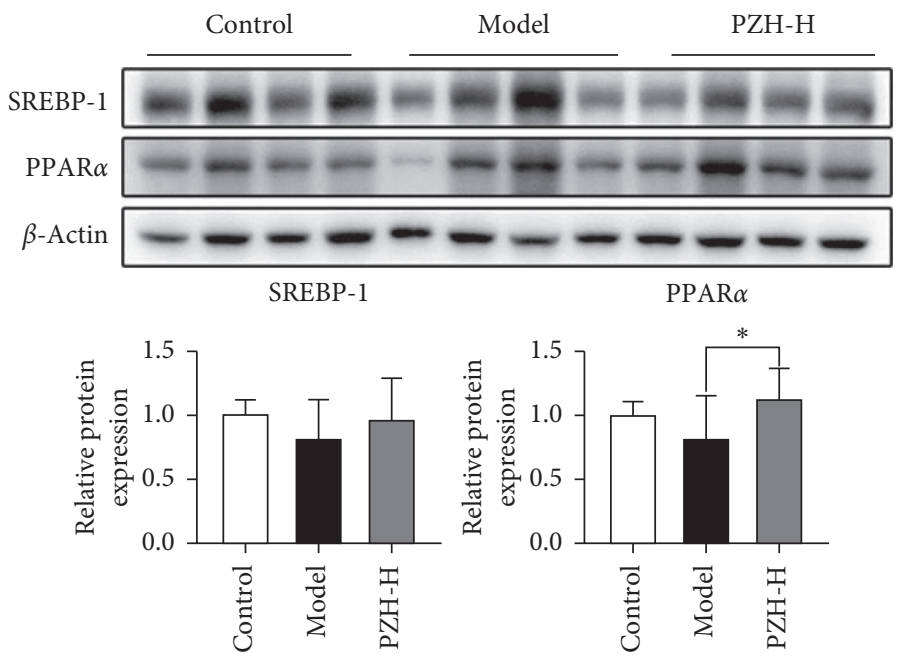

(e)

FIGURe 7: Continued.

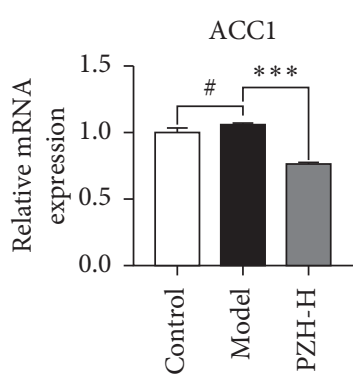

(b)

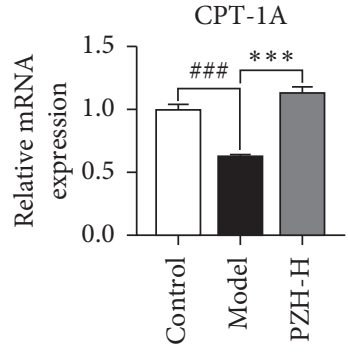

(d)

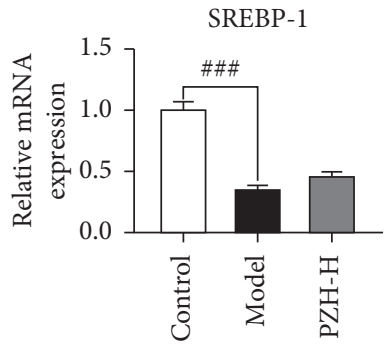




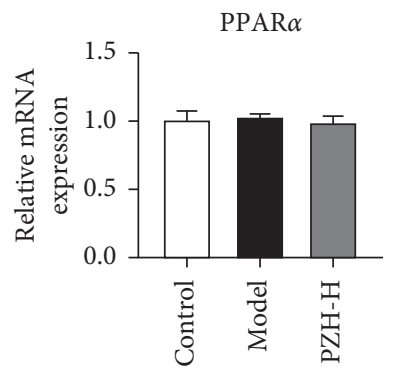

(g)

Figure 7: Effect of PZH on AMPK-ACC-CPT1A pathway. (a) The levels of p-AMPK, AMPK, p-ACC, ACC, and CPT-1A were shown $(n=8)$. The mRNA expression of (b) ACC1, (c) ACC2, and (d) CPT-1A was detected by RT-PCR $(n=8)$. (e) The protein expression of SREBP-1 and PPAR $\alpha$ was shown $(n=8)$. (f) SREBP- 1 mRNA expression and (g) PPAR $\alpha$ mRNA expression were detected $(n=8)$. Data were presented as means \pm SD. ${ }^{\#} P<0.05,{ }^{\# \#} P<0.01$, and ${ }^{\# \# \#} P<0.001$ in the model group vs the control group; ${ }^{*} P<0.05,{ }^{* *} P<0.01$, and ${ }^{* * *} P<0.001$ in the $\mathrm{PZH}-\mathrm{H}$ group vs the model group.

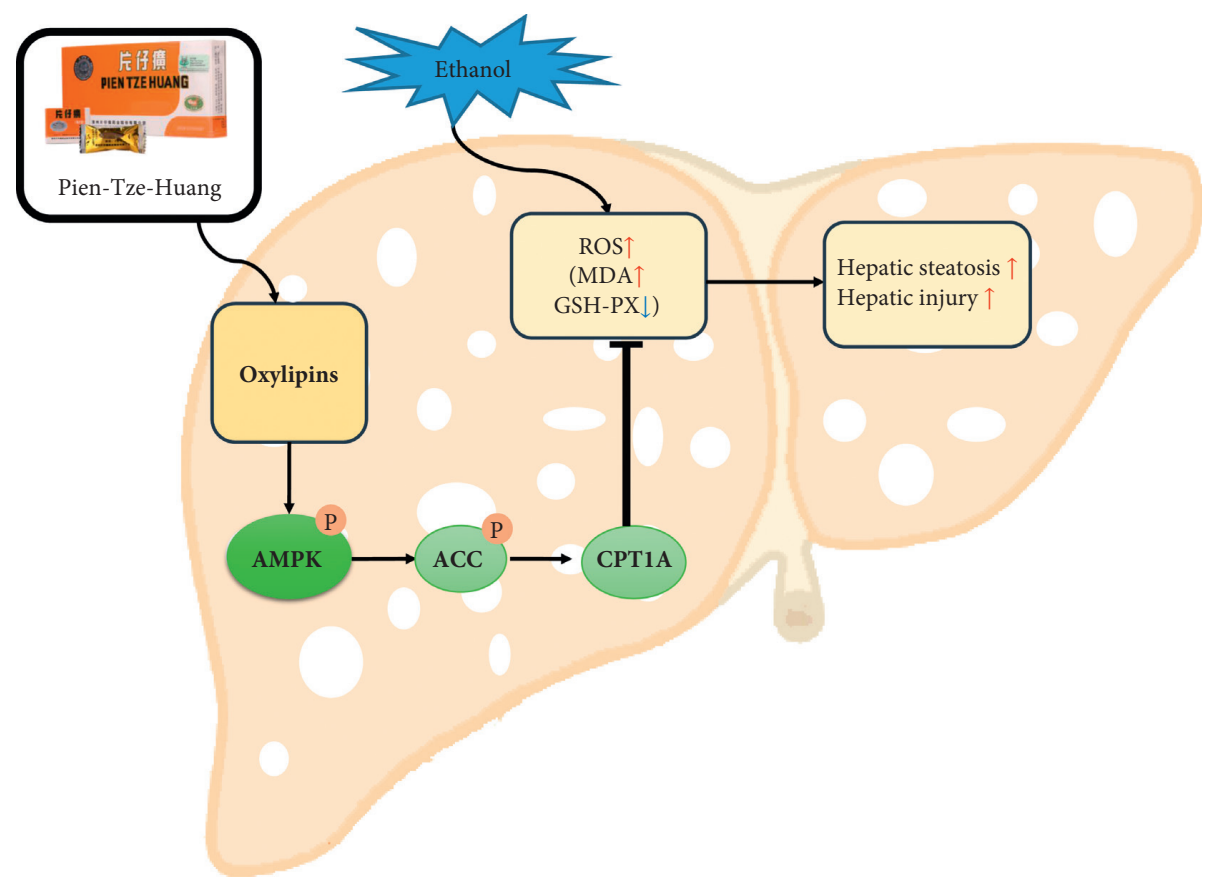

FIGURE 8: Summary of the study. Traditional Chinese formulae PZH increased the levels of oxylipin metabolites, activated AMPK-ACCCPT1A pathway, and finally alleviated oxidative stress and hepatic steatosis.

studies have proved that EPA could improve palmitateinduced endothelial dysfunction and lipotoxicity by activating AMPK pathway $[28,48]$. In addition, LA can reduce hepatic lipid metabolism $[29,30]$, insulin resistance, and adiposity $[49,50]$ via the AMPK pathway. PGE2 can negatively regulate the AMPK pathway $[51,52]$. In this study, we found that PZH increased the levels of EPA and LA and reduced PGE2 levels. In addition, $\mathrm{PZH}$ can activate AMPK and promote the phosphorylation of ACC, which reduces the ACC activity [53] and finally promotes the expression of CPT1A. Although studies have shown that PPAR $\alpha$ and SREBP-1 could improve oxidative stress [54-56], PZH did not regulate their expression in our study, indicating that $\mathrm{PZH}$ did not play a regulatory role in ALD through PPAR $\alpha$ and SREBP-1. However, in this study, we found that the levels of 9-HOTrE, 13-HOTrE, 5,6-dihydroxy-8Z, 11Z, 14Z, 17Z-eicosatetraenoic acid, 17-HETE, and 15-HEPE were increased in the liver of ALD-mice, and $\mathrm{PZH}$ further increased their levels. PGE2 levels were decreased in the liver of ALD-mice, and PZH further decreased this level. We speculated that oxylipin metabolites may need to reach certain concentrations in vivo and activate the AMPK/ACC/ CPT1A pathway to protect against hepatic injury. However, further studies are required to elucidate the underlying mechanism. Although our results showed that PZH could regulate oxylipin metabolites and speculated that Panax notoginseng may mainly enhance oxylipin metabolites, further studies are also needed. 


\section{Conclusions}

In summary, $\mathrm{PZH}$ reduced oxidative stress and alleviated hepatic steatosis and injury. The mechanism was correlated with the oxylipin metabolites/AMPK/ACC/CPT1A pathway (Figure 8).

\section{Data Availability}

The data used to support the findings of this study are available from the corresponding author upon request.

\section{Ethical Approval}

This study (PZSHUTCM200703007) was approved by the Animal Experiment Ethics Committee of Shanghai University of Traditional Chinese Medicine.

\section{Conflicts of Interest}

The authors declare that they have no conflicts of interest.

\section{Authors' Contributions}

Ziye Zhu and Wenjun Zhou contributed equally to this work. Fenghua Li and Yanqi Dang conceived, designed, and supervised the study. Ziye Zhu, Wenjun Zhou, Yang Yang, and Kai Wang performed the experiments. Yanqi Dang, Ziye Zhu, and Wenjun Zhou analyzed the data; and Yanqi Dang and Ziye Zhu wrote the paper. Fenghua Li and Wenjun Zhou revised the manuscript. All authors reviewed and approved the final manuscript.

\section{Acknowledgments}

The authors thank Metabo-Profile R\&D Laboratory (Shanghai, China) for the profiling of oxylipins. This work was supported by the National Natural Science Foundation of China (81804018 and 81808397).

\section{Supplementary Materials}

Supplementary file 1. The levels of oxylipin-metabolites are shown among three groups. Supplementary file 2. 22 and 16 different oxylipin-metabolites are shown in the model group compared with the control group, and the $\mathrm{PZH}-\mathrm{H}$ group compared with the model group, respectively. Supplementary Figure 1. The correlation of oxylipin-metabolites and T-SOD was analyzed by the Spearman correlation analysis. (Supplementary Materials)

\section{References}

[1] J. Rehm and K. D. Shield, "Global burden of alcohol use disorders and alcohol liver disease," Biomedicines, vol. 7, no. 4, 2019.

[2] S. K. Asrani, H. Devarbhavi, J. Eaton, and P. S. Kamath, "Burden of liver diseases in the world," Journal of Hepatology, vol. 70, no. 1, pp. 151-171, 2019.
[3] A. K. Singal, R. Bataller, J. Ahn, P. S. Kamath, and V. H. Shah, "ACG clinical guideline: alcoholic liver disease," The American Journal of Gastroenterology, vol. 113, no. 2, pp. 175-194, 2018.

[4] Y. M. Li and J. G. Fan, "Guidelines of prevention and treatment for alcoholic liver disease (2018, China)," Journal of Digestive Diseases, vol. 20, no. 4, pp. 174-180, 2019.

[5] F. S. Wang, J. G. Fan, Z. Zhang, B. Gao, and H. Y. Wang, "The global burden of liver disease: the major impact of China," Hepatology, vol. 60, no. 6, pp. 2099-2108, 2014.

[6] H. K. Seitz and F. Stickel, "Molecular mechanisms of alcoholmediated carcinogenesis," Nature Reviews Cancer, vol. 7, no. 8, pp. 599-612, 2007.

[7] E. Albano, P. Clot, M. Morimoto, A. Tomasi, M. IngelmanSundberg, and S. W. French, "Role of cytochrome P4502E1dependent formation of hydroxyethyl free radical in the development of liver damage in rats intragastrically fed with ethanol," Hepatology, vol. 23, no. 1, pp. 155-163, 1996.

[8] L. J. Su, J. H. Zhang, H. Gomez et al., "Reactive oxygen species-induced lipid peroxidation in apoptosis, autophagy, and ferroptosis," Oxidative Medicine and Cellular Longevity, vol. 2019, Article ID 5080843, 13 pages, 2019.

[9] Y. Wang, G. Millonig, J. Nair et al., "Ethanol-induced cytochrome P4502E1 causes carcinogenic etheno-DNA lesions in alcoholic liver disease," Hepatology, vol. 50, no. 2, pp. 453-461, 2009.

[10] S. Mueller, T. Peccerella, H. Qin et al., "Carcinogenic etheno DNA adducts in alcoholic liver disease: correlation with cytochrome P-4502E1 and fibrosis," Alcoholism: Clinical and Experimental Research, vol. 42, no. 2, pp. 252-259, 2018.

[11] J. F. Ferguson, K. Roberts-Lee, C. Borcea, H. M. Smith, Y. Midgette, and R. Shah, "Omega-3 polyunsaturated fatty acids attenuate inflammatory activation and alter differentiation in human adipocytes," Journal of Nutritional Biochemistry, vol. 64, pp. 45-49, 2019.

[12] C. Colson, R. A. Ghandour, O. Dufies et al., "Diet supplementation in omega3 polyunsaturated fatty acid favors an anti-inflammatory basal environment in mouse adipose tissue," Nutrients, vol. 11, no. 2, 2019.

[13] C. Hunsche, O. Hernandez, A. Gheorghe, L. E. Diaz, A. Marcos, and M. De la Fuente, "Immune dysfunction and increased oxidative stress state in diet-induced obese mice are reverted by nutritional supplementation with monounsaturated and n-3 polyunsaturated fatty acids," European Journal of Nutrition, vol. 57, no. 3, pp. 1123-1135, 2018.

[14] F. Gottrand, "Long-chain polyunsaturated fatty acids influence the immune system of infants," Journal of Nutrition, vol. 138, no. 9, pp. 1807S-1812S, 2008.

[15] Y. Qu, H. L. Zhang, X. P. Zhang, and H. L. Jiang, "Arachidonic acid attenuates brain damage in a rat model of ischemia/ reperfusion by inhibiting inflammatory response and oxidative stress," Human \& Experimental Toxicology, vol. 37, no. 2, pp. 135-141, 2018.

[16] B. Aydin, Z. Atli Sekeroglu, and V. Sekeroglu, "Effects of whey protein and conjugated linoleic acid on acrolein-induced cardiac oxidative stress, mitochondrial dysfunction and dyslipidemia in rats," Biomedicine \& Pharmacotherapy, vol. 107, pp. 901-907, 2018.

[17] M. Vara-Messler, J. H. Mukdsi, N. I. Osieki et al., "Eicosapentaenoic acid prevents salt sensitivity in diabetic rats and decreases oxidative stress," Nutrition, vol. 72, Article ID 110644, 2020.

[18] J. Peng, J. Xiong, C. Cui et al., "Maternal eicosapentaenoic acid feeding decreases placental lipid deposition and improves the 
homeostasis of oxidative stress through a sirtuin-1 (SIRT1) independent manner," Molecular Nutrition \& Food Research, vol. 63, no. 21, Article ID e1900343, 2019.

[19] K. Palanisamy, R. Krishnaswamy, P. Paramasivan, H. ChihYang, and V. P. Vishwanadha, "Eicosapentaenoic acid prevents TCDD-induced oxidative stress and inflammatory response by modulating MAP kinases and redox-sensitive transcription factors," British Journal of Pharmacology, vol. 172, no. 19, pp. 4726-4740, 2015.

[20] M. V. Simon, D. L. Agnolazza, O. L. German et al., "Synthesis of docosahexaenoic acid from eicosapentaenoic acid in retina neurons protects photoreceptors from oxidative stress," Journal of Neurochemistry, vol. 136, no. 5, pp. 931-946, 2016.

[21] H. Zheng, X. Wang, Y. Zhang, L. Chen, L. Hua, and W. Xu, "Pien-Tze-Huang ameliorates hepatic fibrosis via suppressing NF-kappaB pathway and promoting HSC apoptosis," Journal of Ethnopharmacology, vol. 244, Article ID 111856, 2019.

[22] Y. Yang, Z. Chen, L. Deng et al., "Pien Tze Huang ameliorates liver injury by inhibiting the PERK/eIF2alpha signaling pathway in alcohol and high-fat diet rats," Acta Histochema, vol. 120, no. 6, pp. 578-585, 2018.

[23] Y. Deng, H. Luo, J. Shu et al., "Pien Tze Huang alleviate the joint inflammation in collagen-induced arthritis mice," Chinese Medicine, vol. 15, p. 30, 2020.

[24] L. Huang, Y. Zhang, X. Zhang et al., "Therapeutic potential of pien-tze-huang: a review on its chemical composition, pharmacology, and clinical application," Molecules, vol. 24, no. 18, 2019.

[25] Y. Dang, J. Xu, Y. Yang et al., "Ling-gui-zhu-gan decoction alleviates hepatic steatosis through SOCS2 modification by N6-methyladenosine," Biomedicine \& Pharmacotherapy, vol. 127, Article ID 109976, 2020.

[26] Y. Dang, J. Xu, M. Zhu, W. Zhou, L. Zhang, and G. Ji, “GanJiang-Ling-Zhu decoction alleviates hepatic steatosis in rats by the miR-138-5p/CPT1B axis," Biomedicine \& Pharmacotherapy, vol. 127, Article ID 110127, 2020.

[27] N. Kim, M. S. Kang, M. Nam, S. A. Kim, G. S. Hwang, and H. S. Kim, "Eicosapentaenoic acid (EPA) modulates glucose metabolism by targeting AMP-activated protein kinase (AMPK) pathway," International Journal of Molecular Sciences, vol. 20, no. 19, 2019.

[28] A. Sakamoto, M. Saotome, P. Hasan et al., "Eicosapentaenoic acid ameliorates palmitate-induced lipotoxicity via the AMP kinase/dynamin-related protein-1 signaling pathway in differentiated H9c2 myocytes," Experimental Cell Research, vol. 351, no. 1, pp. 109-120, 2017.

[29] C. Fu, Y. Zhang, Q. Yao et al., "Maternal conjugated linoleic acid alters hepatic lipid metabolism via the AMPK signaling pathway in chick embryos," Poultry Science, vol. 99, no. 1, pp. 224-234, 2020.

[30] T. Y. Zhang, J. T. Huang, H. B. Tian et al., "Trans-10,cis-12 conjugated linoleic acid alters lipid metabolism of goat mammary epithelial cells by regulation of de novo synthesis and the AMPK signaling pathway," Journal of Dairy Science, vol. 101, no. 6, pp. 5571-5581, 2018.

[31] J. Zhao, H. Hu, Y. Wan, Y. Zhang, L. Zheng, and Z. Hong, "Pien Tze Huang Gan Bao ameliorates carbon tetrachlorideinduced hepatic injury, oxidative stress and inflammation in rats," Experimental and Therapeutic Medicine, vol. 13, no. 5, pp. 1820-1826, 2017.

[32] C. Wang, L. Zheng, S. Liu et al., "A novel acidic polysaccharide from the residue of Panax notoginseng and its hepatoprotective effect on alcoholic liver damage in mice,"
International Journal of Biological Macromolecules, vol. 149, pp. 1084-1097, 2020.

[33] F. Liu, X. Bai, R. B. Ding, Y. J. Hu, H. Su, and J. B. Wan, "UPLC/Q-TOFMS-based metabolomics studies on the protective effect of panax notoginseng saponins on alcoholic liver injury," The American Journal of Chinese Medicine, vol. 43, no. 4, pp. 695-714, 2015.

[34] X. Lin, D. Bai, Z. Wei et al., "Curcumin attenuates oxidative stress in RAW264.7 cells by increasing the activity of antioxidant enzymes and activating the Nrf2-Keap1 pathway," PLoS One, vol. 14, no. 5, Article ID e0216711, 2019.

[35] H. C. Hsu, C. Y. Chen, C. H. Chiang, and M. F. Chen, "Eicosapentaenoic acid attenuated oxidative stress-induced cardiomyoblast apoptosis by activating adaptive autophagy," European Journal of Nutrition, vol. 53, no. 2, pp. 541-547, 2014.

[36] F. Echeverria, R. Valenzuela, A. Bustamante et al., "Attenuation of high-fat diet-induced rat liver oxidative stress and steatosis by combined hydroxytyrosol- (HT-) eicosapentaenoic acid supplementation mainly relies on HT," Oxidative Medicine and Cellular Longevity, vol. 2018, Article ID 5109503, 2018.

[37] M. Ebrahimi-Mameghani, H. Jamali, R. Mahdavi, F. Kakaei, R. Abedi, and B. Kabir-Mamdooh, "Conjugated linoleic acid improves glycemic response, lipid profile, and oxidative stress in obese patients with non-alcoholic fatty liver disease: a randomized controlled clinical trial," Croatian Medical Journal, vol. 57, no. 4, pp. 331-342, 2016.

[38] M. Matsuda, T. Kawamoto, and R. Tamura, "Predictive value of serum dihomo-gamma-linolenic acid level and estimated Delta-5 desaturase activity in patients with hepatic steatosis," Obesity Research \& Clinical Practice, vol. 11, no. 1, pp. 34-43, 2017.

[39] T. Inazumi, K. Yamada, N. Shirata et al., "Prostaglandin E2EP4 Axis promotes lipolysis and fibrosis in adipose tissue leading to ectopic fat deposition and insulin resistance," Cell Reports, vol. 33, no. 2, Article ID 108265, 2020.

[40] M. Y. Chung, E. Mah, C. Masterjohn et al., "Green tea lowers hepatic COX-2 and prostaglandin E2 in rats with dietary fatinduced nonalcoholic steatohepatitis," Journal of Medicinal Food, vol. 18, no. 6, pp. 648-655, 2015.

[41] M. Edfawy, M. H. Hassan, A. Mansour, A. A. Hamed, and H. A. Amin, "Meloxicam modulates oxidative stress status, inhibits prostaglandin E2, and abrogates apoptosis in carbon tetrachloride-induced rat hepatic injury," International Journal of Toxicology, vol. 31, no. 3, pp. 276-286, 2012.

[42] W. Wang, L. Yang, L. Song et al., "Combination of Panax notoginseng saponins and aspirin potentiates platelet inhibition with alleviated gastric injury via modulating arachidonic acid metabolism," Biomedicine \& Pharmacotherapy, vol. 134, Article ID 111165, 2021.

[43] J. Yu, W. N. Wang, N. Matei et al., "Ezetimibe attenuates oxidative stress and neuroinflammation via the AMPK/Nrf2/ TXNIP pathway after MCAO in rats," Oxidative Medicine and Cellular Longevity, vol. 2020, Article ID 4717258, 14 pages, 2020.

[44] X. Han, H. Tai, X. Wang et al., "AMPK activation protects cells from oxidative stress-induced senescence via autophagic flux restoration and intracellular NAD (+) elevation," Aging Cell, vol. 15, no. 3, pp. 416-427, 2016.

[45] R. Hu, M. Q. Wang, S. H. Ni et al., "Salidroside ameliorates endothelial inflammation and oxidative stress by regulating the AMPK/NF-kappaB/NLRP3 signaling pathway in AGEs- 
induced HUVECs," European Journal of Pharmacology, vol. 867, Article ID 172797, 2020.

[46] Y. You, Y. L. Liu, Z. Y. Ai et al., "Lactobacillus fermentum KP3-fermented ginseng ameliorates alcohol-induced liver disease in $\mathrm{C} 57 \mathrm{BL} / 6 \mathrm{~N}$ mice through the AMPK and MAPK pathways," Food Function, vol. 11, no. 11, pp. 9801-9809, 2020.

[47] A. Nagappan, J. H. Kim, D. Y. Jung, and M. H. Jung, "Cryptotanshinone from the salvia miltiorrhiza bunge attenuates ethanol-induced liver injury by activation of AMPK/ SIRT1 and Nrf2 signaling pathways," International Journal of Molecular Sciences, vol. 21, no. 1, 2019.

[48] C. H. Lee, S. D. Lee, H. C. Ou, S. C. Lai, and Y. J. Cheng, "Eicosapentaenoic acid protects against palmitic acid-induced endothelial dysfunction via activation of the AMPK/eNOS pathway," International Journal of Molecular Sciences, vol. 15, no. 6, pp. 10334-10349, 2014.

[49] H. Qin, Y. Liu, N. Lu, Y. Li, and C. H. Sun, "Cis-9,trans-11Conjugated linoleic acid activates AMP-activated protein kinase in attenuation of insulin resistance in C2C12 myotubes," Journal of Agricultural and Food Chemistry, vol. 57, no. 10, pp. 4452-4458, 2009.

[50] S. Jiang, Z. Wang, J. J. Riethoven, Y. Xia, J. Miner, and M. Fromm, "Conjugated linoleic acid activates AMP-activated protein kinase and reduces adiposity more effectively when used with metformin in mice," Journal of Nutrition, vol. 139, no. 12, pp. 2244-2251, 2009.

[51] C. Wang, Y. Gao, Z. Zhang et al., "Safflower yellow alleviates osteoarthritis and prevents inflammation by inhibiting PGE2 release and regulating NF-kappaB/SIRT1/AMPK signaling pathways," Phytomedicine, vol. 78, Article ID 153305, 2020.

[52] K. Funahashi, X. Cao, M. Yamauchi, Y. Kozaki, N. Ishiguro, and F. Kambe, "Prostaglandin E2 negatively regulates AMPactivated protein kinase via protein kinase a signaling pathway," Prostaglandins \& Other Lipid Mediators, vol. 88, no. 1-2, pp. 31-35, 2009.

[53] Y. Minokoshi, Y. B. Kim, O. D. Peroni et al., "Leptin stimulates fatty-acid oxidation by activating AMP-activated protein kinase," Nature, vol. 415, no. 6869, pp. 339-343, 2002.

[54] H. Yaribeygi, M. T. Mohammadi, and A. Sahebkar, "PPARalpha agonist improves hyperglycemia-induced oxidative stress in pancreatic cells by potentiating antioxidant defense system," Drug Research, vol. 68, no. 6, pp. 355-360, 2018.

[55] Y. Zhou, W. Yang, Z. Li et al., "Moringa oleifera stem extract protect skin keratinocytes against oxidative stress injury by enhancement of antioxidant defense systems and activation of PPARalpha," Biomedicine \& Pharmacotherapy, vol. 107, pp. 44-53, 2018.

[56] A. Sharma, S. K. Anand, N. Singh, U. N. Dwivedi, and P. Kakkar, "Berbamine induced AMPK activation regulates mTOR/SREBP-1c axis and Nrf2/ARE pathway to allay lipid accumulation and oxidative stress in steatotic HepG2 cells," European Journal of Pharmacology, vol. 882, Article ID 173244, 2020. 